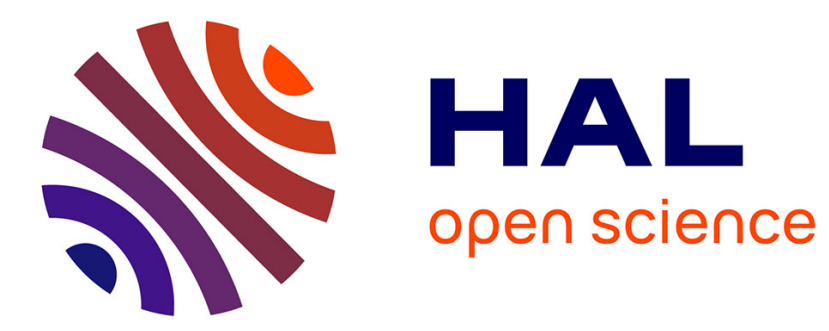

\title{
Pansharpening of images acquired with color filter arrays
}

Daniele Picone, Mauro Dalla Mura, Laurent Condat

\section{To cite this version:}

Daniele Picone, Mauro Dalla Mura, Laurent Condat. Pansharpening of images acquired with color filter arrays. SPIE Photonics Europe 2018, Apr 2018, Strasbourg, France. hal-01851522

\section{HAL Id: hal-01851522 \\ https://hal.science/hal-01851522}

Submitted on 30 Jul 2018

HAL is a multi-disciplinary open access archive for the deposit and dissemination of scientific research documents, whether they are published or not. The documents may come from teaching and research institutions in France or abroad, or from public or private research centers.
L'archive ouverte pluridisciplinaire HAL, est destinée au dépôt et à la diffusion de documents scientifiques de niveau recherche, publiés ou non, émanant des établissements d'enseignement et de recherche français ou étrangers, des laboratoires publics ou privés. 


\title{
Pansharpening of images acquired with color filter arrays
}

\author{
Daniele Picone, Mauro Dalla Mura, Laurent Condat ${ }^{\mathrm{a}}$ \\ ${ }^{a}$ Univ. Grenoble Alpes, CNRS, Grenoble INP*, GIPSA-lab, 38000 Grenoble, France \\ ${ }^{*}$ Institute of Engineering Univ. Grenoble Alpes
}

\begin{abstract}
In remote sensing, a common scenario involves the simultaneous acquisition of a panchromatic (PAN), a broadband high spatial resolution image, and a multispectral (MS) image, which is composed of several spectral bands but at lower spatial resolution. The two sensors mounted on the same platform can be found in several very high spatial resolution optical remote sensing satellites for Earth observation (e.g., Quickbird, WorldView and SPOT)

In this work we investigate an alternative acquisition strategy, which combines the information from both images into a single band image with the same number of pixels of the PAN. This operation allows to significantly reduce the burden of data downlink by achieving a fixed compression ratio of $1 /\left(1+b / \rho^{2}\right)$ compared to the conventional acquisition modes. Here, $b$ and $\rho$ denote the amount of distinct bands in the MS image and the scale ratio between the PAN and MS, respectively (e.g.: $b=\rho=4$ as in many commercial high spatial resolution satellites). Many strategies can be conceived to generate such a compressed image from a given set of PAN and MS sources. A simple option, which will be presented here, is based on an application of the color filter array (CFA) theory. Specifically, the value of each pixel in the spatial support of the synthetic image is taken from the corresponding sample either in the PAN or in a given band of the MS upsampled to the size of the PAN. The choice is deterministic and done according to a custom mask. There are several works in the literature proposing various methods to construct masks which are able to preserve as much spectral content as possible for conventional RGB images. However, those results are not directly applicable to the case at hand, since it deals with i) images with different spatial resolution, ii) potentially more than three spectral bands and, iii) in general, different radiometric dynamics across bands. A tentative approach to address these issues is presented in this work.

The compressed image resulting from the proposed acquisition strategy will be processed to generate an image featuring both the spatial resolution of the PAN and the spectral bands of the MS. This final product allows a direct comparison with the result of any standard pansharpening algorithm; the latter refers to a specific instance of data fusion (i.e., fusion of a PAN and MS image), which differs from our scenario since both sources are separately taken as input. In our setting, the fusion step performed at the ground segment will jointly involve a fusion and reconstruction problem (also known as demosaicing in the CFA literature). We propose to address this problem with a variational approach.

We present in this work preliminary results related to the proposed scheme on real remote sensed images, tested over two different datasets acquired by the Quickbird and Geoeye-1 platforms, which show superior performances compared to applying a basic radiometric compression algorithm to both sources before performing a pansharpening protocol. The validation of the final products in both scenarios allows to visually and numerically appreciate the tradeoff between the compression of the source data and the quality loss suffered.
\end{abstract}

Keywords: Image fusion, data compression, remote sensing, inverse problems, color filter arrays

\section{INTRODUCTION}

With the ever-increasing availability of remote sensors monitoring the Earth surface, the scientific literature has shown that image fusion can effectively combine information from different imaging sources. ${ }^{1}$ By combining

Further author information: (Send correspondence to D.P.)

D.P.: E-mail: daniele.picone@gipsa-lab.grenoble-inp.fr, Telephone: +33 (0)4 76574355

M.D.M.: E-mail: mauro.dalla-mura@gipsa-lab.grenoble-inp.fr, Telephone: +33 (0)4 76826482

L.C.: E-mail: laurent.condat@gipsa-lab.grenoble-inp.fr, Telephone: +33 (0)4 76826492 


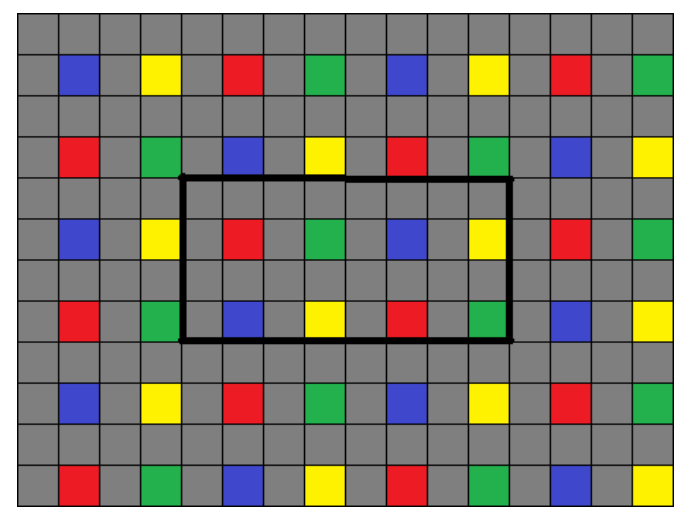

Figure 1. Periodic mask for CFA-based compression described in section 2.2. In the figure gray dots are assigned to the PAN image, while each of the four color are assigned to each the four bands of the MS (typically RGB and NIR)

complementary multisensor, multitemporal and/or multiview information into one new image it is possible to generate results with a quality that could not be achieved otherwise. ${ }^{2}$ Pansharpening is a specific instance of this problem, aimed at combining a PAN and a MS image for generating a synthetic image with highest possible spatial and spectral resolution, ${ }^{3}$ as they aren't achievable simultaneously with a single sensor because of physical constraints. Such products are in high demand both in commercial venues, e.g. by Google Earth and Bing Maps, and as pre-processing step for other applications, such as change detection, ${ }^{4}$ object recognition, ${ }^{5}$ visual image analysis and scene interpretation. ${ }^{6}$ Pansharpening techniques proposed by the literature ${ }^{7,8}$ range from simple approaches $^{7}$ to more advanced variational models. ${ }^{9,10}$

In this paper, we propose a novel acquisition scheme of PAN and MS in which the two multiresolution images are combined into a single compressed acquisition. Indeed, with the availability of lower budget small satellite carrying high-quality optical imagery, ${ }^{11}$ on-board image compression has became an increasingly interesting field to compensate for limited on-board resources in terms of mass memory and downlink bandwidth. Many strategies have been developed to deal with this issue, giving focus on ease of on-board implementation, both through software $^{12}$ and optical devices, such as the Coded Aperture Snapshot Spectral Imaging (CASSI), ${ }^{13}$ a compressive acquisition system based on the shifted superimposition of masked hyperspectral spectral components.

The contribution of this work is twofold: i) we present a compression scheme tailored on multisensor sources with cheap on-board hardware implementation with optical devices, inspired by the theory of Color Filter Arrays (CFA), ${ }^{14}$ ii) we present an inversion scheme capable of dealing simultaneously with the problem of decompression and reconstruction of fused product of such sources, confirming the effectiveness of classical regularization approaches for image processing such as Total Variation (TV). ${ }^{15}$

\section{ACQUISITION AND INVERSION MODEL}

\subsection{Notation}

We will assume that every matrix, denoted with a bold uppercase variable, can be also represented by the corresponding lowercase letter when in lexicographic order (by concatenating each column into a single vector). In particular, the original source is composed of a wide-band PAN $\mathbf{P} \in \mathbb{R}^{n_{p 1} \times n_{p 2}}$ and a MS $\mathbf{M} \in \mathbb{R}^{n_{m 1} \times n_{m 2} \times n_{b}}$, where $n_{b}$ represents the amount of bands to sharpen in the MS. The spatial scale ratio $\rho$ between the PAN and the MS is assumed to be the same in cross-track and along-track direction, so that the total number of pixel $n_{p}=n_{p 1} n_{p 2}$ and $n_{m}=n_{m 1} n_{m 2}$ of the PAN and MS respectively are related by $n_{m}=n_{p} / \rho^{2}$. The set of bands of the MS will be denoted by $\left\{\mathbf{M}_{k}\right\}_{k=1, \ldots, n_{b}}$ and their upscaled version by $\left\{\tilde{\mathbf{M}}_{k}\right\}_{k=1, \ldots, n_{b}}$. Additionally, the $[. ;$.$] and [.,$.$] operators respectively stand for column and row concatenation, while o denotes the Hadamard$ (element-wise) product. 


\subsection{Description of CFA-based compression}

The compression scheme proposed in this work will aim at generating a synthetic image $\mathbf{Y} \in \mathbb{R}^{n_{p 1} \times n_{p 2}}$, which embeds information both from the PAN and MS sources and exactly matches the size of the PAN source, hence achieving a compression ratio $r=\rho^{2} /\left(n_{b}+\rho^{2}\right)$. In the optimal scenario, this compressed acquisition should be able to retain as much information as possible for the reconstruction of the sharpened result. We envision to realize this compression scheme with ease of optical implementation in mind, as this procedure to be done on-board exclusively with optical hardware devices, to be placed on cascade to the classical acquisition sensors. This leads to choosing compression systems that are only capable of mostly straightforward computation; for the sake of convenience, as most optical devices can be modeled as linear systems, we will assume that all on-board operations are linear and we will present a tentative scheme for an optical implementation.

In this work, we focus in particular on a method based on Color Filter Arrays (CFA): In the context of photography, CFAs define a matrix of color filters placed on a grid of photo-sensors, chosen from a subset of possible spectral responses. Mathematically, if we consider an incident source with a certain spectral radiance $L(\lambda)$, the incident radiance $L_{i n c}$ of an element of this matrix can be obtained as

$$
L_{i n c}=\int L(\lambda) \xi_{k}(\lambda) d \lambda
$$

where $\xi_{k}(\lambda)$ is the spectral response of the $k$-th color filter, chosen within an assigned set $\{\xi(\lambda)\}_{k=1, \ldots, n_{b}}$, which has a bandpass behavior on ideally non-overlapping wavelengths. If we suppose to add a color filter array to a matrix of MS sensors, this operation can be seen as masking. Specifically, if we assume that our acquisition system were capable of acquiring every spectral response for all pixels, then we can select only one for each pixel through an operation of binary masking (detailed in the next section). In greater detail, we propose in this work to generate the compressed image $\mathbf{Y}$ such that the value of each pixel in its spatial support is taken from the corresponding sample either in the PAN or in a given band of the upsampled MS, according to the following equation:

$$
\mathbf{Y}=\mathbf{P} \circ \mathbf{H}_{0}+\sum_{k=1}^{n_{b}} \tilde{\mathbf{M}}_{k} \circ \mathbf{H}_{k}
$$

where $\left\{\mathbf{H}_{k}\right\}_{k=0, \ldots, n_{b}}$ is a set of binary masks (made up of just 0 and 1 ) that do not share any non-zero value for each spatial position. Stacking those masks into a 3D matrix allows for a representation as a color-coded map: an unique color is assigned to each available band (both from the PAN or the MS), and the pixels they are in charge of are colored accordingly. As the interpolated sources $\mathbf{M}_{k}$ is usually generated by software through an upsampling and a spatial degradation by a low-pass filter, it would be useful to only transfer original unprocessed

MS samples to $\mathbf{Y}$. Consequently, we assign all the pixels introduced by the extension in $\tilde{\mathbf{M}}_{k}$ to active (transparent to the passage of light) values of the PAN mask $\mathbf{H}_{0}$. Regarding the rest of the pixels, a reasonable distribution of the assignments to each MS band should target saving as much spectral information as possible, which is the same target of many studies on optimal CFA, especially in the case of 3 bands. ${ }^{16}$ The cited work, in particular, suggests a criterion of rejection of maximum distance, which for four bands would generate the mask shown in figure 1. This compression scheme can be represented as a linear transformation, through the formulation:

$$
\mathbf{y}=\mathbf{C}_{0} \mathbf{p}+\sum_{k=1}^{n_{b}} \mathbf{C}_{k} \tilde{\mathbf{m}}_{k}=\mathbf{C}[\mathbf{p} ; \tilde{\mathbf{m}}]
$$

where $\mathbf{C}_{k}=\operatorname{diag}\left(\mathbf{h}_{k}\right)$ and $\mathbf{C}$ is a block matrix, whose blocks are made up of all the matrices $\left\{\mathbf{C}_{k}\right\}_{k=0, \ldots, n_{b}}$.

\subsection{Physical implementation}

A physical prototype of the proposed scheme could be done in various configurations; in principle, the ideal solution would involve a matrix of acquisition sensors exactly matching the CFA structure shown in figure 1. Unfortunately, PAN and MS sensors generally operate with different technologies, as they are optimized to overcome complementary physical constraints: MS sensors acquire more energy from larger ground areas, while PAN sensors from wider bandwidths of wavelengths. For a matrix of exclusively MS sensors, the CFA could be implemented with optical filters realized with patterned optical coatings, made possible by recent advances in 


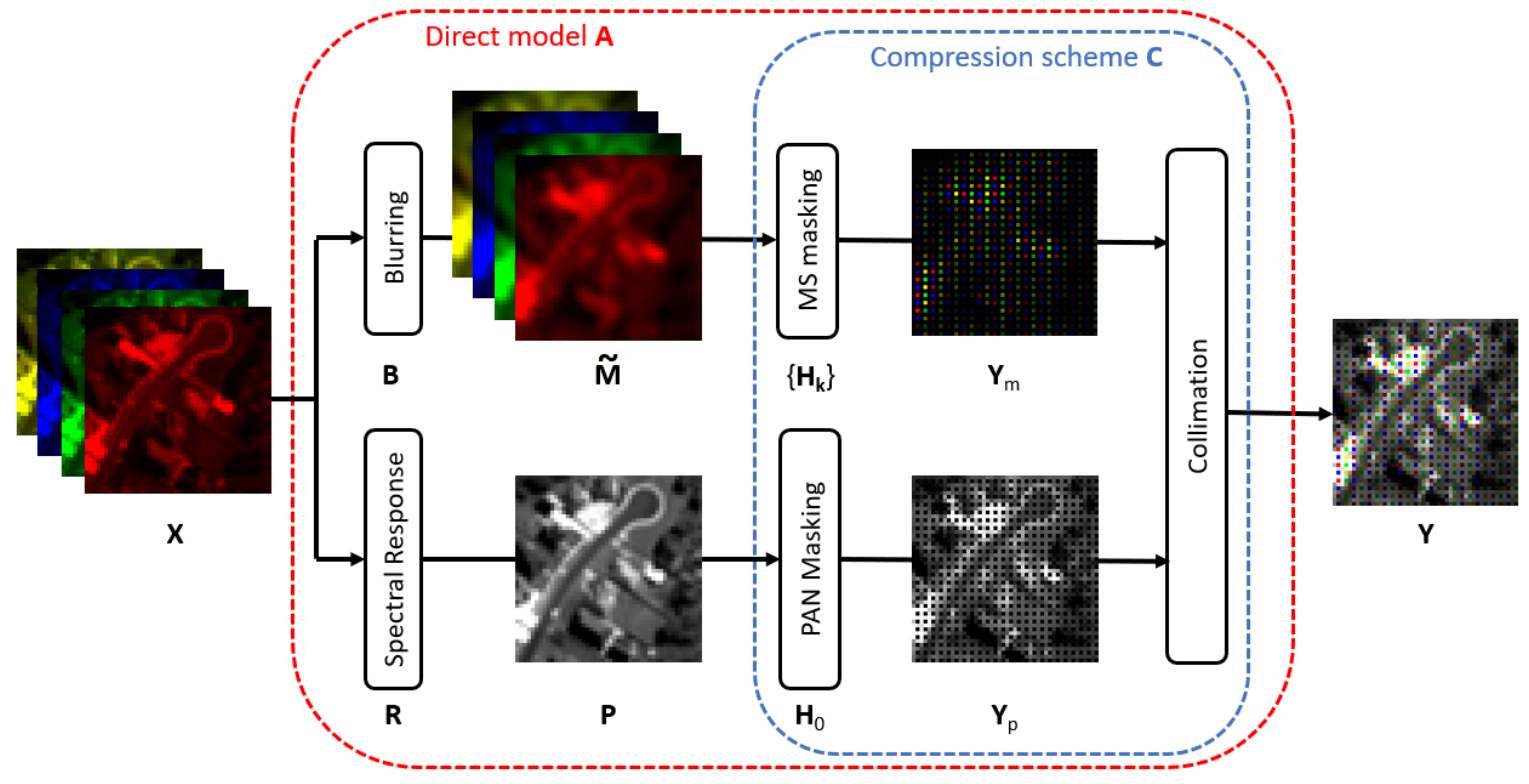

Figure 2. Direct model for reconstruction of the fused image from the compressed acquisition; pure black pixels in $\mathbf{Y}_{p}$ and $\mathbf{Y}_{m}$ are shut off by masking

micro-lithography and coating technologies. ${ }^{17}$ When considering a classical setup, such as in the configuration in which PAN and MS sensors are separate (and with potentially distinct optical paths), the implementation could be done by separating each MS component with a dispersive element, usually a prism, and let each component pass through an assigned coded aperture, which is in charge of ideally realizing the operation of masking described in eq. 2. The PAN signal could go through a similar optical processing, with a coded aperture that could implement $\mathbf{H}_{0}$; the PAN sensor could also intentionally feature holes in the places where the acquisition is not needed, avoiding the redundancy of acquisition and the need of a mask. The results for each of those operations can be collimated appropriately on Focal Plane Array (FPA) detector, which is in charge of integrating the post-processed scene to generate $\mathbf{Y}$. It is worth noting that the reconstruction method (which will be described below) does not require that the position of each processed sample is the same as described in section 2.2; any permutation of the samples is allowed, as long as the final position of each is known beforehand by the ground segment. That implies that the two sources can be kept separate and managed by two different FPAs, if the collimation would pose any challenge in the implementation.

\subsection{Reconstruction scheme}

We propose to solve the problem of reconstruction, or demosaicing as commonly known in the CFA literature, through a variational scheme. Specifically, we will make use of an inversion scheme for the estimation of an ideal unknown target image $\mathbf{X} \in \mathbb{R}^{n_{p 1} \times n_{p 2} \times n_{b}}$, which features both the spatial resolution of the PAN and spectral resolution of the MS. Specifically, our target is to invert the scheme shown in figure 2. The ideal signal is supposed to generate the PAN and the interpolated MS through the following system:

$$
\left\{\begin{array}{l}
\tilde{\mathbf{m}}=\mathbf{B x}+\mathbf{e}_{m} \\
\mathbf{p}=\mathbf{R x}+\mathbf{e}_{p}
\end{array}\right.
$$

where $\mathbf{B} \in \mathbb{R}^{n_{p} n_{b} \times n_{p} n_{b}}$ is the the blurring of the MS sensor and $\mathbf{R} \in \mathbb{R}^{n_{p} \times n_{p} n_{b}}$ the spectral response of the MS sensor relative to the one of PAN sensor. $\mathbf{e}_{m}$ and $\mathbf{e}_{p}$ are the error models, which will be statistically characterized as independent instances of additive white Gaussian noise with zero mean. Subsequently, $\mathbf{P}$ and $\tilde{\mathbf{M}}$ are processed as described in section 2.2 to obtain the final compressed acquisition $\mathbf{y}$, which is transmitted to the ground segment; the latter in charge of generating an estimation of $\mathbf{x}$, which will be denoted by $\hat{\mathbf{x}}$. This 


\begin{tabular}{|c|c|c|c|c|c|c|c|c|}
\hline & \multicolumn{4}{|c|}{ San Francisco } & \multicolumn{4}{|c|}{ Hobart } \\
\hline & ERGAS & SAM & Q4 & $\mathrm{sCC}$ & ERGAS & SAM & Q4 & $\mathrm{sCC}$ \\
\hline Ideal value & 0 & 0 & 1 & 1 & 0 & 0 & 1 & 1 \\
\hline EXP & 5.260 & 2.505 & 0.9264 & 0.5465 & 6.446 & 3.025 & 0.8819 & 0.5162 \\
\hline MTF-GLP-CBD & 3.988 & 2.750 & 0.9565 & 0.7216 & 4.311 & 3.079 & 0.9420 & 0.7171 \\
\hline$\overline{\mathbf{C F}} \overline{\mathbf{F}} \overline{\mathbf{A}} \overline{\mathbf{T}} \overline{\mathbf{V}}$ & $\overline{5.978}$ & $3.9 \overline{1} \overline{0}$ & $\overline{0} . \overline{9} \overline{36}$ & $0.5 \overline{8} \overline{3}$ & $\overline{6} . \overline{8} 1 \overline{5}$ & $4.5 \overline{0}$ & $\overline{0} \overline{8} \overline{723}$ & $\overline{0.517 \overline{6}}$ \\
\hline BINNING & 9.024 & 6.395 & 0.8136 & 0.4606 & 6.153 & 4.403 & 0.8416 & 0.5362 \\
\hline
\end{tabular}

Table 1. Reduced resolution validation for the Hobart and San Francisco datasets. Best results for compressed sources (below dashed line) in bold

inversion will be treated as a variational problem; in other words, the estimation is realized through the following minimization:

$$
\hat{\mathbf{x}}=\arg \min _{\mathbf{x}^{\prime}}\left\|\mathbf{A} \mathbf{x}^{\prime}-\mathbf{y}\right\|_{2}^{2}+\lambda \phi\left(\mathbf{x}^{\prime}\right)
$$

where $\mathbf{A}=\mathbf{C}[\mathbf{B} ; \mathbf{R}], \phi: \mathbb{R}^{n_{p} n_{b}} \rightarrow \mathbb{R}^{+}$is a scalar function, called regularizer, and $\lambda$ is a user-chosen scalar which weights each of the two contributes. It is worth noticing that, contrary to the usual decompression schemes, this method doesn't focus on the reconstruction of the original sources $\mathbf{P}$ and $\tilde{\mathbf{M}}$, but tries to directly estimate a signal which simultaneously features information from both sources. Various strategies can be implemented for the regularization; one widespread option is the total variation, ${ }^{15}$ which in this work is used in its anisotropic form:

$$
\phi(\mathbf{x})=\sum_{k=1}^{n_{b}} \sum_{i, j}\left(\left|\Delta_{x} \mathbf{X}_{k}\{i, j\}\right|+\left|\Delta_{y} \mathbf{X}_{k}\{i, j\}\right|\right)
$$

where $\Delta_{x}$ and $\Delta_{y}$ denote the discrete gradients in the vertical and horizontal direction, respectively, and $\{i, j\}$ indicates the spatial position they are computed at.

\section{EXPERIMENTAL RESULTS}

Two datasets will be considered in the experiments; they are both composed of a PAN image and a 4-band MS with a scale of 1:4. The San Francisco dataset features a residential American area and acquired by the Quickbird platform (PAN sizes: $1024 \times 1024$ pixels at spatial resolution of $0.6 \mathrm{~m}$ ). The Hobart dataset was acquired by the GeoEye-1 satellite (PAN sizes: $2048 \times 2048$ pixels at spatial resolution of $0.5 \mathrm{~m}$ ) and represents an moderately urban area in Tanzania.

For the objective quality assessment, we employ the reduced resolution validation, according to the Wald's protocol. ${ }^{18}$ Specifically, the original MS image will work as reference (or ground truth - GT); the latter and the original PAN image are degraded with filters matching their Modulation Transfer Function (MTF) and taken as sources to generate the sharpened image. This product is then compared with the GT through a set of quality indices; in particular, we consider the Spectral Angle Mapper (SAM), Erreur Relative Globale Adimensionnelle de Synthese (ERGAS), the $Q_{4}$ index and the spatial Cross Correlation (sCC). ${ }^{7}$

The following preliminary tests are aimed mostly as proof of concept for the applicability of our model. To deal with the different dynamics of multimodal sources, we pre-processed the PAN signal to match his mean and variance with the ones of $\mathbf{q}=\mathbf{R} \tilde{\mathbf{m}}$, which is a simulation of the PAN obtained with the information from the MS, following the histogram matching procedure typical of component substitution fusion algorithms, ${ }^{3}$ obtaining:

$$
\mathbf{P}_{\mathbf{p p}}=\left(\mathbf{P}-\mu_{P}\right) \frac{\sigma_{q}}{\sigma_{P_{L}}}+\mu_{q}
$$

where $\mu_{P}$ and $\mu_{q}$ denote the mean of $\mathbf{P}$ and $\mathbf{q}$ respectively, $\sigma_{q}$ and $\sigma_{P_{L}}$ are the standard deviations respectively of $\mathbf{q}$ and $\mathbf{P}_{\mathbf{L}}$, a version of the PAN degraded to the resolution of the MS.

Tests are conducted at reduced resolution, assuming a scale ratio of $\rho=2$; the degraded sources were fused with the best performing classical protocol to assess the expected performances when no compression step is provided. The best results were achieved with the Generalized Laplacian Pyramid with MTF-matched filter and regression based injection model (MTF-GLP-CBD). ${ }^{7}$ The interpolation of the MS data (EXP) is performed with 


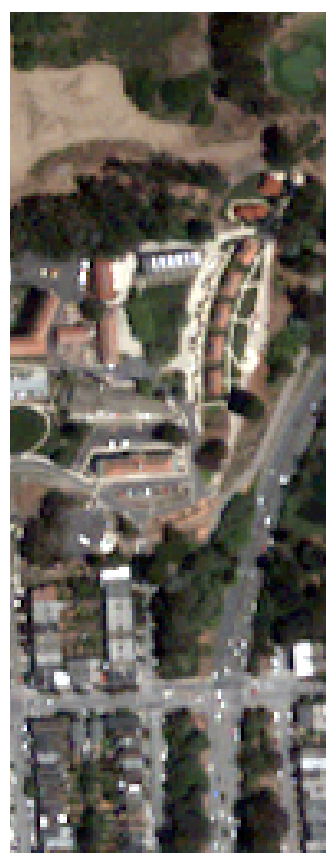

(a) GT

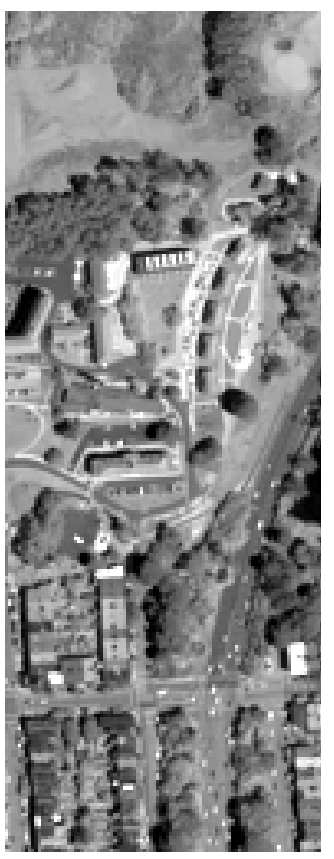

(b) PAN

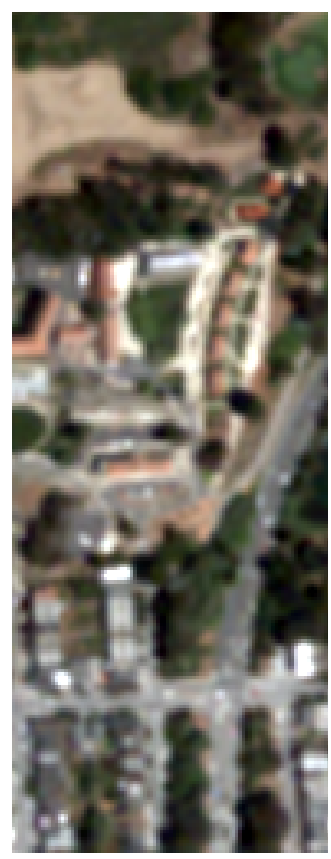

(c) EXP

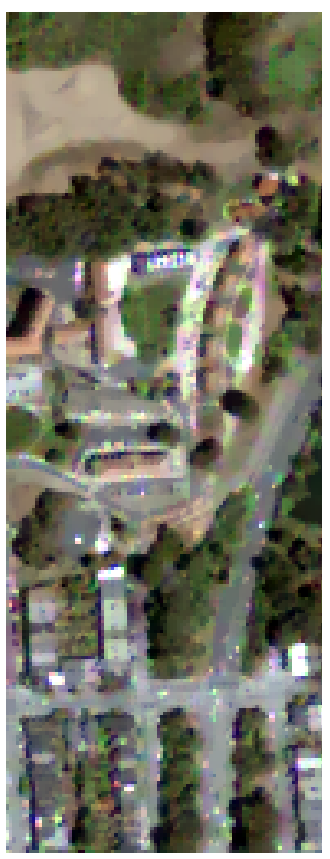

(d) $\mathrm{CFA}+\mathrm{TV}$

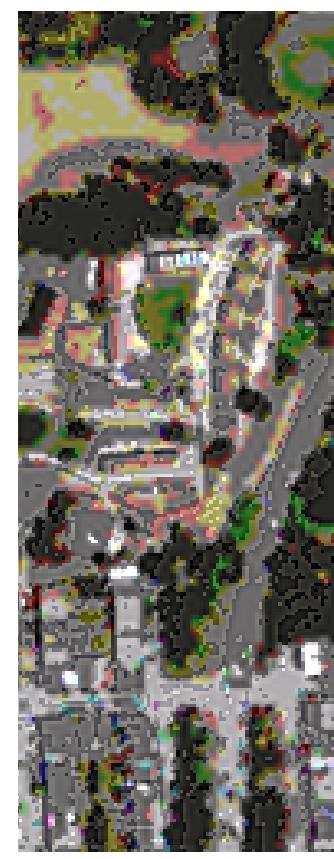

(e) Binning

Figure 3. Visual comparison of selected tests described in section 3 on the San Francisco dataset (detail)

a 23-tap Lagrange polynomial filter. ${ }^{19}$ In order to have a fair comparison, we have setup an experiment which achieve the same compression ratio. Specifically, we feature a test involving a compression based on binning of quantization levels; if the acquired signal is originally represented with $L$ bits, hence with $2^{L}$ quantization levels, the compression will reduce the number of quantization levels to $\left\lceil 2^{L^{\prime}}\right\rceil$ by uniformly grouping adjacent levels together. The choice of $L^{\prime}$, which we suppose generally non integer for simplicity of comparison regardless of its practical meaning, is imposed by the compression ratio $r=2^{L} / 2^{L^{\prime}}$ that we want to achieve. This procedure is applied to both the MS and the PAN, which are then fused with the MTF-GLP-CBD method.

With regards to our proposed method, after the CFA-based compression, the inversion through the total variation regularization was solved with the dual-primal algorithm, ${ }^{20}$ where $\lambda$ was selected by analyzing the best Q4 outcome.

Table 1 shows the results of the reduced resolution quality assessment, while a visual comparison for the Hobart dataset is provided in fig. 3. The objective analysis does not show a drastic reduction in performance compared to the classical pansharpening methods with no compression step, as long as an appropriate combination of on-board acquisition and regularization is selected. The CFA-based compression with the inversion based on $\mathrm{TV}$ is the best option in terms of spatial quality (for which $\mathrm{sCC}$ is a good indicator); radiometric binning has a devastating effect on the fusion process, which are mostly evident in the visual analysis, as many areas are patched together because of binning.

\section{CONCLUSIONS AND FUTURE PERSPECTIVES}

In this work, we proposed a model to jointly address the problem of image fusion and reconstruction of compressed data; a particular focus is given on ease of implementation with optical components on board of low-budget satellites, with could justify mass production of a constellation of the latter. The relaxed constraints on downlink resources is paid at a cost of a more complex and demanding processing on the ground segment. Preliminary tests have shown the potential of the proposed approach in preserving the quality in the reconstructed image by employing a reconstruction technique based on total variation. Future investigation will involve confrontation with other compression schemes proposed by the literature, both hardware, such as CASSI, and software; inter- 
esting insights could also be obtained by investigating an optimal compression scheme, which produces the best fused result with a given compression ratio or to improve the hardware requirements for the implementation.

\section{REFERENCES}

[1] Thomas, C., Ranchin, T., Wald, L., and Chanussot, J., "Synthesis of multispectral images to high spatial resolution: A critical review of fusion methods based on remote sensing physics," IEEE Trans. Geosci. Remote Sens. 46, 1301-1312 (May 2008).

[2] Dalla Mura, M., Prasad, S., Pacifici, F., Gamba, P., Chanussot, J., and Benediktsson, J. A., "Challenges and opportunities of multimodality and data fusion in remote sensing," Proc. IEEE 103, 1585-1601 (Sept 2015).

[3] Alparone, L., Aiazzi, B., Baronti, S., and Garzelli, A., [Remote Sensing Image Fusion], CRC Press (2015).

[4] Souza, Jr., C., Firestone, L., Silva, L. M., and Roberts, D., "Mapping forest degradation in the Eastern Amazon from SPOT 4 through spectral mixture models," Remote Sens. Environ. 87, 494-506 (Nov. 2003).

[5] Mohammadzadeh, A., Tavakoli, A., and Valadan Zoej, M. J., "Road extraction based on fuzzy logic and mathematical morphology from pan-sharpened IKONOS images," Photogramm. Rec. 21, 44-60 (Mar. 2006).

[6] Laporterie-Déjean, F., de Boissezon, H., Flouzat, G., and Lefèvre-Fonollosa, M.-J., "Thematic and statistical evaluations of five panchromatic/multispectral fusion methods on simulated PLEIADES-HR images," Inform. Fusion 6, 193-212 (Sep. 2005).

[7] Vivone, G., Alparone, L., Chanussot, J., Dalla Mura, M., Garzelli, A., Licciardi, G., Restaino, R., and Wald, L., "A critical comparison among pansharpening algorithms," IEEE Trans. Geosci. Remote Sens. 53, 25652586 (May 2015).

[8] Loncan, L., Fabre, S., Almeida, L. B., Bioucas-Dias, J. M., Wenzhi, L., Briottet, X., Licciardi, G. A., Chanussot, J., Simoes, M., Dobigeon, N., Tourneret, J. Y., Veganzones, M. A., Qi, W., Vivone, G., and Yokoya, N., "Hyperspectral pansharpening: A review," IEEE Geosci. Remote Sens. Mag. 3, 27-46 (Sep. 2015).

[9] Bioucas-Dias, J. and Figueiredo, M., "A new TwIST: Two-step iterative shrinkage/thresholding algorithms for image restoration," IEEE Transactions on Image Processing 16, 2992-3004 (dec 2007).

[10] Wright, S., Nowak, R., and Figueiredo, M., "Sparse reconstruction by separable approximation," IEEE Transactions on Signal Processing 57, 2479-2493 (jul 2009).

[11] Yu, G., Vladimirova, T., and Sweeting, M. N., "Image compression systems on board satellites," Acta Astronautica 64, 988-1005 (may 2009).

[12] Huang, B., ed., [Satellite Data Compression], Springer New York (2011).

[13] Arce, G. R., Brady, D. J., Carin, L., Arguello, H., and Kittle, D. S., "Compressive coded aperture spectral imaging: An introduction," IEEE Signal Processing Magazine 31, 105-115 (jan 2014).

[14] Nakamura, J., [Image Sensors and Signal Processing for Digital Still Cameras (Optical Science and Engineering)], CRC Press (2005).

[15] Rudin, L. I., Osher, S., and Fatemi, E., "Nonlinear total variation based noise removal algorithms," Physica D: Nonlinear Phenomena 60(1-4), 259-268 (1992).

[16] Condat, L., "A new random color filter array with good spectral properties," in [2009 16th IEEE International Conference on Image Processing (ICIP)], IEEE (nov 2009).

[17] Eichenholz, J. M. and Dougherty, J., "Ultracompact fully integrated megapixel multispectral imager," in [Integrated Optics: Devices, Materials, and Technologies XIII], Broquin, J.-E. and Greiner, C. M., eds., SPIE (feb 2009).

[18] Wald, L., Ranchin, T., and Mangolini, M., "Fusion of satellite images of different spatial resolutions: Assessing the quality of resulting images," Photogramm. Eng. Remote Sens. 63, 691-699 (Jun. 1997).

[19] Aiazzi, B., Alparone, L., Baronti, S., and Garzelli, A., "Context-driven fusion of high spatial and spectral resolution images based on oversampled multiresolution analysis," IEEE Trans. Geosci. Remote Sens. 40, 2300-2312 (Oct. 2002).

[20] Combettes, P. L. and Pesquet, J.-C., "Primal-dual splitting algorithm for solving inclusions with mixtures of composite, lipschitzian, and parallel-sum type monotone operators," Set-Valued and Variational Analysis $\mathbf{2 0}$, 307-330 (aug 2011). 